Systematic Review

\title{
What Are the Predictors of Altered Central Pain Modulation in Chronic Musculoskeletal Pain Populations? A Systematic Review
}

Jacqui Clark, MSc ${ }^{1,2,3}$, Jo Nijs, PhD ${ }^{2,3}$, Gillian Yeowell, PhD' , and Peter Charles Goodwin, PhD'

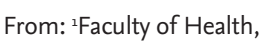
Psychology and Social Care, Manchester Metropolitan University, Manchester, United Kingdom; ${ }^{2}$ Faculty of Physical Education and Physiotherapy, Vrije Universiteit Brussel, Brussels, Belgium: ${ }^{3}$ Pain in Motion international research collaboration, Brussels, Belgium

Address Correspondence: Jacqui Clark, MSc

Dept. of Physiotherapy

Manchester Metropolitan University

53 Bonsall St, Birley Fields Manchester, UK M15 6GX E-mail: jclark@thephysioshed.com

Disclaimer: There was no external funding in the preparation of this manuscript.

Conflict of interest: Each author certifies that he or she, or a member of his or her immediate family, has no commercial association

(i.e., consultancies, stock ownership, equity interest, patent/licensing arrangements, etc.) that might pose a conflict of interest in connection with the submitted manuscript.

Manuscript received: 01-25-2017 Accepted for publication: 03-13-2017

Free full manuscript: www.painphysicianjournal.

com
Background: Altered central pain modulation is the predominant pain mechanism in a proportion of chronic musculoskeletal pain disorders and is associated with poor outcomes. Although existing studies predict poor outcomes such as persistent pain and disability, to date there is little consensus on what factors specifically predict altered central pain modulation.

Objectives: To review the existing literature on the predictive factors specifically for altered central pain modulation in musculoskeletal pain populations.

Study Design: This is a systematic review in accordance with supplemented PRISMA guidelines.

Methods: A systematic search was performed by 2 mutually blinded reviewers. Relevant articles were screened by title and abstract from Medline, Embase, PubMed, CINAHL, and Web of Science electronic databases. Alternative sources were also sought to locate missed potential articles. Eligibility included studies published in English, adults aged 18 to 65, musculoskeletal pain, baseline measurements taken at the pre-morbid or acute stage, $>3$-month follow-up time after pain onset, and primary outcome measures specific to altered central pain modulation. Studies were excluded where there were concurrent diseases or they were non-predictive studies. Risk of bias was assessed using the quality in prognostic studies (QUIPS) tool. Study design, demographics, musculoskeletal region, inclusion/ exclusion criteria, measurement timelines, predictor and primary outcome measures, and results were extracted. Data were synthesized qualitatively and strength of evidence was scored using the grading of recommendations, assessment, development, and evaluations (GRADE) scoring system.

Results: Nine eligible articles were located, in various musculoskeletal populations (whiplash, $\mathrm{n}=$ 2; widespread pain, $n=5$; temporomandibular disorder, $n=2$ ). Moderate evidence was found for 2 predictive factors of altered central pain modulation: 1) high sensory sensitivity (using genetic testing or quantitative sensory tests), and 2) psychological factors (somatization and poor self-expectation of recovery), at a pre-morbid or acute stage baseline.

Limitations: At the times of the article publications, the current definitions and clinical guidelines for identifying altered central pain modulation were not yet available. Careful interpretation of the information provided using current knowledge and published guidelines was necessary to extract information specific to altered central pain modulation in some of the studies, avoiding unwarranted assumptions.

Conclusions: Premorbid and acute stage high sensory sensitivity and/or somatization are the strongest predictors of altered central pain modulation in chronic musculoskeletal pain to date. This is the first systematic review specifically targeting altered central pain modulation as the primary outcome in musculoskeletal pain populations. Early identification of people at risk of developing chronic pain with altered central pain modulation may guide clinicians in appropriate management, diminishing the burden of persistent pain on patients and heath care providers alike.

Systematic Review Registration no.: PROSPERO 2015:CRD42015032394.

Key words: Predictive factors, pre-morbid and acute stage baselines, altered central pain modulation, chronic musculoskeletal pain, sensory processing, somatization

Pain Physician 2017; 20:487-500 
hronic pain is experienced when, subsequent to the subacute phase of healing, pain persists beyond the expected healing timeframe, leading to poor outcomes. Existing studies have investigated predictors of poor outcomes associated with musculoskeletal pain, including disability $(1,2)$ and failure to return to work $(3,4)$. However, there remains little consensus, probably due to the heterogeneity of outcomes studied and, moreover, the heterogeneity of pain mechanisms. Hence, the transition from acute musculoskeletal pain to chronic pain is currently difficult to predict.

Common to a significant proportion of chronic musculoskeletal pain populations is the phenomenon of sensitization of the central nervous system pain pathways, i.e., altered central pain modulation. Altered central pain modulation manifests as a predominantly non-nociceptive, non-neuropathic pain mechanism $(5,6)$ and is defined as a dysregulation of the central nervous system causing neuronal hyper-excitability, characterized by generalized hypersensitivity of the somatosensory system to both noxious and non-noxious stimuli $(5,7)$. Altered central pain modulation involves impaired modulatory mechanisms within the central nervous system whereby nociceptive pathways are less inhibited and nociceptive facilitatory pathways enhanced, resulting in augmentation of nociceptive transmission (8).

Poor outcomes such as disability are not necessarily an indication of altered central pain modulation per se, despite being commonly associated with each other $(9,10)$. Disability may be the result of psychological factors that may not be predominantly a result of altered central pain modulation, such as fear avoidance (11). Similarly, poor outcomes such as chronic pain may or may not be an indication of altered central pain modulation, depending on the predominant pain mechanism. It is proposed that the phenomenon of altered central pain modulation should be investigated specifically in the etiology of poor outcomes.

A strong clinical predictor of altered central pain modulation is "disproportionate, non-mechanical, unpredictable pattern of pain provocation in response to multiple/non-specific aggravating/easing factors" (5). Altered central pain modulation is associated with many non-specific chronic musculoskeletal pain conditions and the etiology is poorly understood. It is considered by some that altered central pain modulation is a disease in itself rather than a disease of the particular presenting musculoskeletal condition (12). From a clini- cal perspective, identifying predictors of altered central pain modulation may help to sub-classify "at-risk" patients at baseline after acute musculoskeletal pain onset. Appropriate management could then be prioritized accordingly to minimize the risk of altered central pain modulation and poor outcomes.

Therefore, the objective of the current study is to systematically evaluate the current available literature to identify predictors of altered central pain modulation in adults with general musculoskeletal pain conditions, measured at a pre-morbid or acute stage baseline and followed up at least 3 months later. Furthermore, if data allow, predictors for altered central pain modulation specifically in patients with non-specific low back pain (LBP) might be identified as a second objective.

The scope of the current review follows the type of model intended to inform clinicians' therapeutic decision-making, in accordance with Moons et al (13). It intends to focus on prognostic studies designed to predict a future health outcome (altered central pain modulation) as opposed to diagnostic predictor models or models designed to identify a suspected existing disease (13).

\section{Methods}

The review protocol was registered prior to commencement of the search with PROSPERO, protocol no.: PROSPERO 2015:CRD42015032394. The methods used in the current study follow the guidelines published in the PRISMA Statement for systematic reviews (14). This is supplemented by methodological guidelines specific to systematic reviews of prognostic studies by Dretzke et al (15) and Moons et al (13).

\section{Search Strategy}

The following electronic databases were searched from their inception up to March 2016: EMBASE (via Ovid), Medline (via Ovid), CINAHL (EBSCO), Scopus, Web of Science (via Web of Knowledge), and Google Scholar. Reference lists of the eligible studies were hand searched and 31 other researchers in the field were contacted by email by JC in order to identify any missed, potentially important studies.

A pilot search was carried out to test preliminary search terms identified from related literature. With a view to finding studies detailing prognostic indicators which predict altered central pain modulation, the search term "Prognos*" was piloted. This was with the intention of capturing terms such as prognosis, prognostic indicators, prognostic factors, poor prognosis, 
and was initially focused on LBP populations. However, it became clear that the studies with chronic LBP and prognos* were generally looking at the natural course of LBP or the response to management regarding whether or not they would return to work. Therefore, the pilot search was altered to acute LBP AND prognos* because this would potentially yield prognostic indicators for a poor outcome in acute LBP. However, poor outcome in acute LBP can lead to various outcomes such as disability or persistent pain, which are not specific to altered central pain modulation. Therefore, specific terms for the outcome measures of altered central pain modulation had to be developed, with the assistance of examples drawn from other review studies in altered central pain modulation $(16,17)$.

The term predict* was chosen because statistically logistic regression models are used to find predictors (18). Dretzke et al (15) advise the use of both prognosis- and predictor-related terms, without filters, so as to minimise loss of relevant studies.

The term "central sensitisation" was also piloted. It became clear that there are 2 spellings, English and American, the latter using " $z$," as in "sensitization." Both spellings had to be included. No word filters were applied to the search strategy.

Subsequently, the systematic search was conducted to locate studies relevant to 3 key subject areas of the research question: 1) central sensitization pain due to altered central pain modulation, 2) predictors, and 3) musculoskeletal pain known to be associated with altered central pain modulation (19), using the tested search terms. Keywords or database specific search terms (e.g., MeSH, subject terms, subject headings, and CINAHL headings) or a combination of both were used. The Boolean operators "OR" and "AND" were used to combine search terms within and between each of the subject areas. No time limits were applied to any of the databases. No filters were used in the search strategies, as recommended by Dretzke et al (15). Only full text studies reported in English were to be included. The systematic search was carried out independently by JC and PG. The search terms are detailed in Table 1.

\section{Eligibility Criteria}

The review included only predictive or prognostic studies where baseline predictive factor measurements were taken pre-morbidly or at the acute stage of musculoskeletal pain onset. The primary outcome measurements were those that indicate a likelihood of the pain mechanism being specific to altered central pain modulation, measured at least 3 months after the initial acute pain onset. Longitudinal data were used in logistic regression models of analysis to identify predictors of altered central pain modulation.

Although prognostic longitudinal cohort studies using logistic regression models of analysis were expected in the search, it was agreed at the outset not to restrict the search to those only using logistic regression models of analysis. This decision was made in anticipation of a small number of studies eligible for inclusion to avoid unnecessary exclusion. It was proposed, a priori, that authors of potentially relevant studies could be contacted for permission to re-run their data through a logistic regression analysis if necessary and if possible.

Of critical importance to this review was the primary outcomes specific to altered central pain modulation. An anticipated potential difficulty was the lack of a single gold standard measurement tool for the determination of altered central pain modulation. Quantitative sensory testing (QST) is an acceptable measurement procedure for sensory hypersensitivity (20), a manifestation of altered central pain modulation. Another acceptable measure of altered central pain modulation is the Central Sensitisation Inventory

Table 1. Search terms.

\begin{tabular}{|c|c|}
\hline \multicolumn{2}{|l|}{ Target Population: } \\
\hline Musculoskeletal pain & $\begin{array}{l}\text { ("low back pain" OR backache OR lumbago OR "ache, low back" OR "Low back pain" OR "neck } \\
\text { pain" OR "cervical pain*” OR cervicalgia OR cervicodynia OR "temporomandibular pain "*” OR } \\
\text { "widespread pain" OR "musculoskeletal pain" OR "shoulder pain" OR whiplash) }\end{array}$ \\
\hline \multicolumn{2}{|l|}{ Target condition: } \\
\hline $\begin{array}{l}\text { Central sensitisation pain; altered central } \\
\text { pain modulation }\end{array}$ & $\begin{array}{l}\text { ("Central pain" OR "central sensitisation" OR "central sensitization" OR "central sensitivity" OR } \\
\text { "central hypersensitivity" OR "endogenous analgesia" OR "descending nociceptive inhibition" OR } \\
\text { "descending facilitation" OR "nociceptive facilitation" OR "central pain modulation") }\end{array}$ \\
\hline \multicolumn{2}{|l|}{ Methodology: } \\
\hline $\begin{array}{l}\text { Prospective predictive cohort studies using } \\
\text { regression analysis }\end{array}$ & $\begin{array}{l}\text { (inception OR prognos }{ }^{\star} \text { OR predict* OR prospective OR cohort OR longitudinal OR "follow-up" } \\
\text { OR "follow up study" OR Risk) }\end{array}$ \\
\hline
\end{tabular}


(CSI) questionnaire (21) validated in 2013 by Neblett and colleagues (22). The CSI gives a score that indicates the likelihood of symptoms being attributed to altered central pain modulation. More recent clinical guidelines have been available detailing how to clinically identify altered central pain modulation $(5,7)$. Outcome measurements paralleling any of these guidelines were anticipated as being acceptable in the search process, especially for studies published before 2012, which did not use QST as the primary outcome measure.

\section{Study Selection}

Studies were screened according to titles and then by abstracts, based on the inclusion and exclusion criteria listed in Table 2. All studies were independently screened by 2 reviewers (JC/PG) before collaboration on the screening. In the case of disagreement, a third reviewer was available for consultation (GY). Discussion between reviewers enabled a consensus to be reached regarding the eligibility of the final studies for inclusion.

\section{Risk of Bias (quality) Assessment}

At the study level, the Quality in Prognostic Studies (QUIPS) (23) risk of bias tool for prognostic studies was used to assess the quality of each study. This was tailored to the requirements of the review and supplemented by recommendations from the Checklist for Critical Appraisal and Data Extraction for Systematic Reviews of Prediction Modelling Studies (CHARMS) checklist (13). The final 7-part risk of bias checklist was used to grade each study with an overall score of low, moderate, or high risk of bias, according to the QUIPS grading guidelines. The risk of bias grades were taken into consideration when evaluating the strength of findings in each predictive study.

Overall quality of evidence and strength of recommendation was determined using the Grading of Recommendations, Assessment, Development and Evaluations (GRADE) criteria (24). The final GRADE score incorporated the 4 categories: quality, consistency, directness, and effect size. Evidence quality was based on the overall GRADE scores for each comparison and graded: high (at least 4 points overall), moderate (3 points), low (2 points), or very low (1 or less).

\section{Data Extraction (Selection and Coding)}

$J C$ and PG independently extracted results from the included studies. A data extraction form was agreed upon between the 2 reviewers prior to extraction based on the required information and research question. Authors were contacted directly by email in the event of data queries.

The data extracted were study design, characteristics of participants (number/age/gender/musculoskeletal pain condition), recruitment setting, inclusion/exclusion criteria, baseline time point since injury, primary outcome measure, predictive factor measures, followup time points, and main findings. The main findings varied in their summary measures.

Given the small number of studies and the variation in predictors and outcome measures across the selection, statistical pooling of data was not feasible. Instead, findings were synthesized qualitatively.

\section{Results}

The initial search yield was 2,368 hits from the databases and 13 from additional sources (Fig. 1). After removal of duplicates, 171 articles were selected from the initial hits. Screening of the titles, using the inclusion and exclusion criteria reduced the yield to 107 . Further screening by abstract reduced the yield to 36 . One article was excluded as it could not be retrieved (25). Further exclusions were made based on nonEnglish language reporting $(n=2)$, primary outcomes not specific to altered central pain modulation $(n=$ $22)$, too short a follow-up time $(n=1)$, patients being above age $65(n=1)$, and only associations being calculated $(n=1)$. The total number of full articles

Table 2. Eligibility criteria for study screening.

\begin{tabular}{|l|l||}
\hline \multicolumn{1}{|c||}{ Inclusion Criteria } & \multicolumn{1}{c|}{ Exclusion criteria } \\
\hline Prognostic longitudinal studies & Non musculoskeletal pain populations \\
\hline Participants - Adult (age 18 - 65) & People aged under 18 or over 65 \\
\hline $\begin{array}{l}\text { Recruited pre-morbidly or at the acute pain onset with follow-up at } \\
\text { least 3 months after pain onset }\end{array}$ & Specific pathologies; post-surgical pain studies \\
\hline $\begin{array}{l}\text { Musculoskeletal pain (known to be associated with altered central } \\
\text { pain modulation) }\end{array}$ & $\begin{array}{l}\text { Rheumatoid arthritis or any other rheumatic, neurological, } \\
\text { oncological or internal disease }\end{array}$ \\
\hline $\begin{array}{l}\text { Measuring an outcome of altered central pain modulation according } \\
\text { to clinical guidelines (if described) or using QST }\end{array}$ & $\begin{array}{l}\text { Functional outcomes not specific to altered central pain modulation } \\
\text { such as return to work or disability-only outcomes }\end{array}$ \\
\hline
\end{tabular}




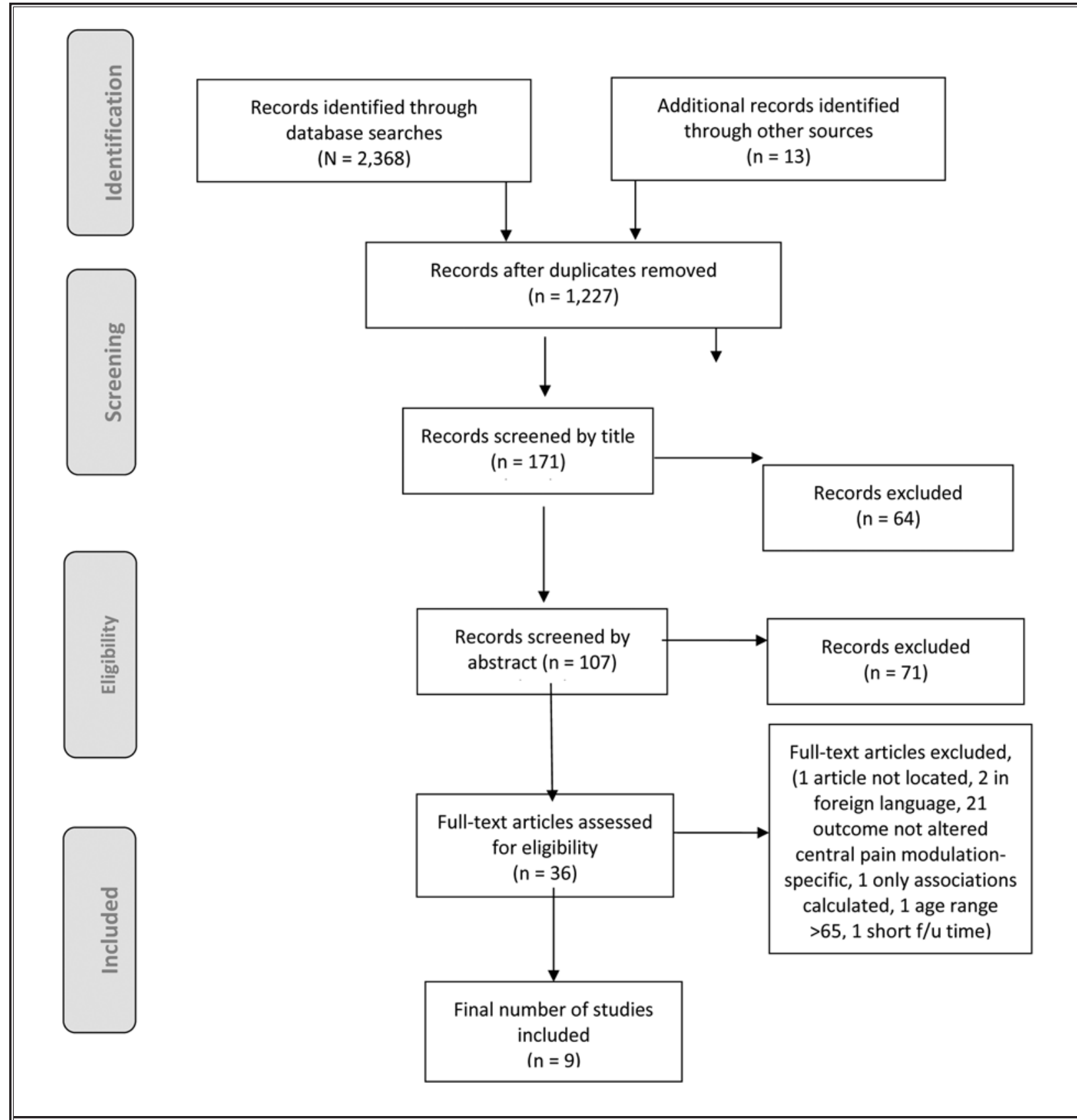

Fig. 1. PRISMA flowchart describing the selection of articles.

selected was 9. Full text articles were screened by JC and PG and there was no disagreement requiring consultation with the third reviewer (GY). Based on the research question, the inclusion and exclusion criteria, and clinical knowledge of altered central pain modulation, it was agreed by consensus that 9 studies meet study eligibility $(9,10,26-32)$.
One corresponding author was contacted to clarify a reporting error - the study reported that high tender point counts significantly predict widespread pain (WP) but quoted a non-significant $P$ value of 0.157 (29). It was confirmed by the author as a typographical error in the article and corrected as $P=0.042$. The study demographics are summarized in Table 3. 
Table 3. Study demographics.

\begin{tabular}{|c|c|c|c|}
\hline Study & Age (years) & Male / Female & Setting \\
\hline McBeth et al (26) 2001 & Range $=18-64$ & $\begin{array}{l}\text { Male } \mathrm{n}=608 \\
\text { Female } \mathrm{n}=796\end{array}$ & Random population sample, UK \\
\hline Sterling et al (9) 2003 & $\begin{array}{l}\text { Mean }=36.27(\mathrm{SD}+/-12.69) \\
\text { Controls: mean }=40.1 \\
\quad(\mathrm{SD}+/-13.6 \text { years })\end{array}$ & $\begin{array}{c}\text { Male } \mathrm{n}=24, \\
\text { Female } \mathrm{n}=56 \\
20 \text { controls } \\
8 \text { males, } 12 \text { females }\end{array}$ & $\begin{array}{l}\text { Hospital accident and emergency departments, } \\
\text { primary care practices (medical and physiotherapy) and } \\
\text { media advertisements }\end{array}$ \\
\hline Harkness et al (27) 2004 & Median $=23$ & Male Approx. 1/3 & 12 diverse occupational settings \\
\hline $\begin{array}{l}\text { Diatchenko et al (28) } \\
2005\end{array}$ & Range $=18-34$ & Females $\mathrm{n}=202$ & $\begin{array}{l}\text { Setting not mentioned } \\
\text { ? population study implied }\end{array}$ \\
\hline $\begin{array}{l}\text { Wynne-Jones et al (30) } \\
2006\end{array}$ & $\begin{array}{c}\text { Median }=41 \text { yrs. }[\mathrm{IQR}= \\
33-50]\end{array}$ & Female $=51 \%$ & UK based vehicle insurance co. \\
\hline Gupta et al (29) 2007 & $\begin{array}{l}25-39 n=66(28.6 \%) \\
40-49 n=54(23.4 \%) \\
50-65 n=111(48 \%)\end{array}$ & $\begin{aligned} \text { Male } \mathrm{n} & =71(30.7 \%) \\
\text { Female } \mathrm{n} & =160(69.3 \%)\end{aligned}$ & $\begin{array}{l}\text { Three population-based primary care registers covering } \\
\text { two socio-demographically mixed suburban areas }\end{array}$ \\
\hline Ferrari (10) 2010 & Mean $=37.5(S D+/-13)$ & $\begin{array}{l}\text { Male } \mathrm{n}=32 \\
\text { Female } \mathrm{n}=37\end{array}$ & Single primary care walk-in clinic in Canada \\
\hline Slade et al (31) 2014 & Range $=18-44$ & Not stated & $\begin{array}{l}\text { OPPERA (Orofacial Pain: Prospective Evaluation and Risk } \\
\text { Assessment) research clinic USA }\end{array}$ \\
\hline Markkula et al (32) 2016 & Mean $=27.7(S D \pm 7.3)$ & $\begin{array}{c}\text { Male }=46.2 \% \\
\text { Female }=53.8 \%\end{array}$ & Finnish Twin Cohort, Finland \\
\hline
\end{tabular}

\section{Study Characteristics}

All the studies were prospective longitudinal cohort studies (Table 4), all investigated prognostic factors with an outcome measure related to altered central pain modulation.

Baseline measurements of predictors were taken pre-morbidly by the majority of studies $(26-29,31,32)$ and at the acute stage of the pain in the other studies $(9,10,30)$, so that it was likely that baseline predictors were measured before the onset of altered central pain modulation. Follow-up measurements were all taken at time points beyond the normal healing timeframe, ranging from 3 months $(10,28)$ to 6 months $(9), 12$ months (26,30), 15 months (29), 24 months (27), 5 years (31), and 15 years (32).

Predictors varied widely across studies and can be grouped according to sensory sensitivity, psychological, and other factors. Six studies $(9,26,28,29,31,32)$ used sensory sensitivity at baseline as a predictive factor of altered central pain modulation. Diatchenko and colleagues (28) specifically used a genetic marker for sensitivity, unlike the others which included QST or the American College of Rheumatology (ACR) (33) criteria as predictors. Psychological measures included Somatising Symptoms Checklist $(26,29)$, Illness Attitudes Scale $(26,30)$, Illness Behaviour Score (29), Recovery Expectation Questionnaire (10), perception of pre-morbid general health including psychological distress, using the General Health Questionnaire $(9,2630)$, and workrelated psychosocial risk factors (27). Work-related physical factors (27) and collision-specific factors (30) were also tested as predictors.

\section{Risk of Bias Assessment}

All studies were judged as low risk of bias (Table 5). The study by Diatchenko and colleagues (28) initially presented as high risk of bias. It was written in a style relevant to its background of genetics and according to the journal requirements in which it was published and in order to review it fairly, the supporting information was obtained from the journal website.

Three main groups of predictors were identified across the studies as: 1) sensory sensitivity factors, 2) psychological factors, and 3) other factors (Table 6). According to the groups, there might be a higher risk of the patient developing altered central pain modulation, if:

- High sensory sensitivity can be identified at baseline using QST or the ACR guidelines for tender point counts or genetic testing for sensory sensitivity;

- Somatization, poor illness attitudes, and negative expectation of recovery can be identified at baseline (Somatisation Checklist; Illness Attitudes questionnaire; Expectation of Recovery questionnaire);

- Pre-morbid frequent headaches were apparent. 
Predictors of Altered Central Pain Modulation in Chronic Musculoskeletal Pain

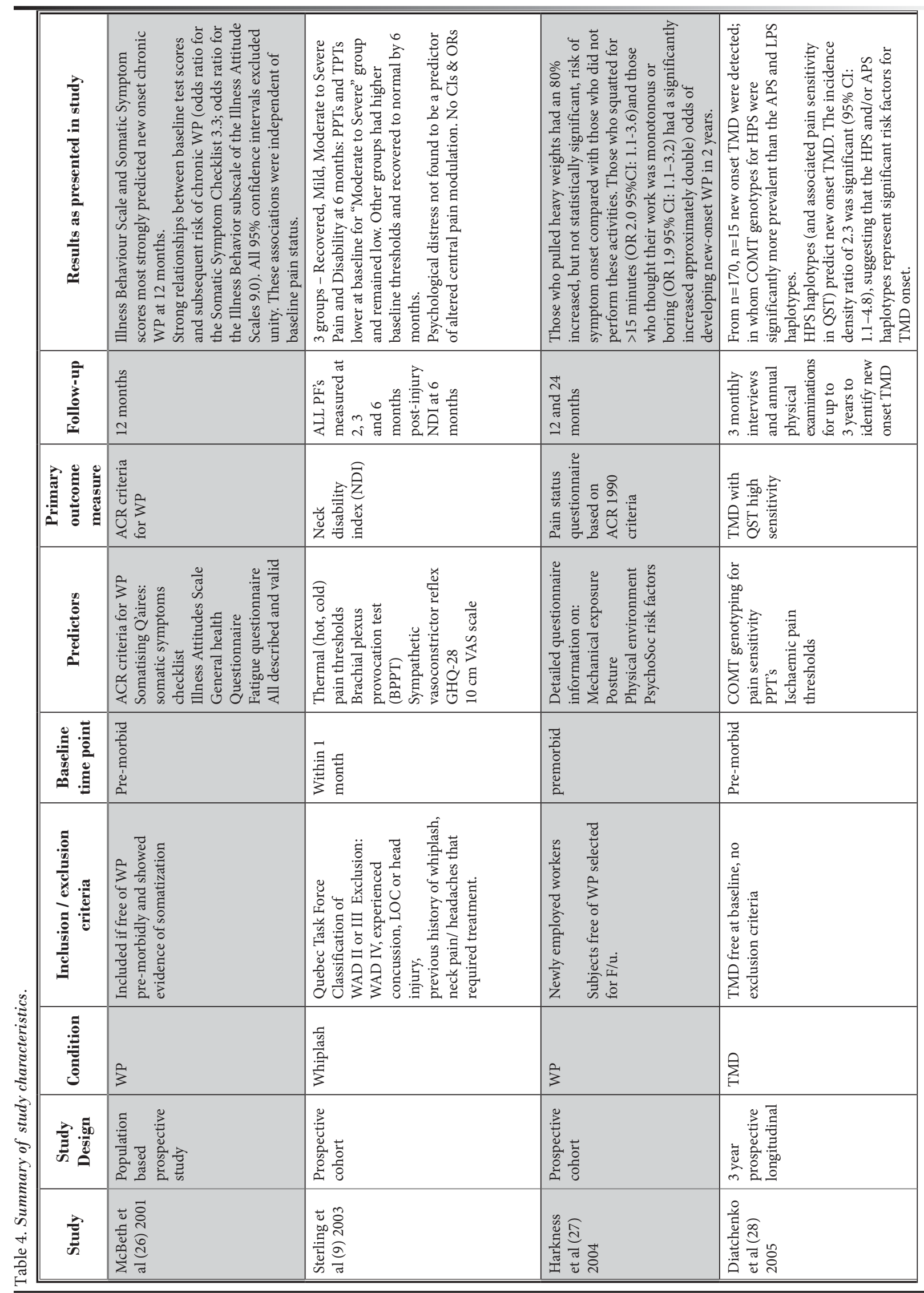


Pain Physician: September/October 2017: 20:487-500

\begin{tabular}{|c|c|c|c|c|c|}
\hline & 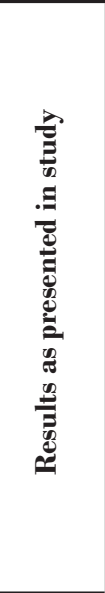 & 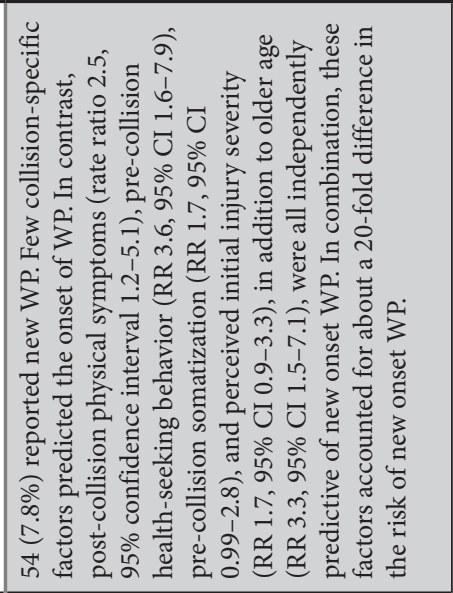 & 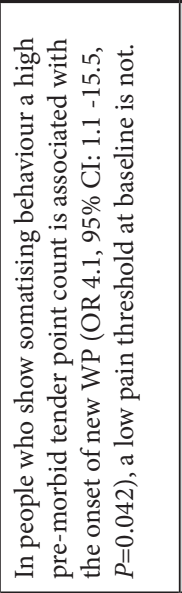 & 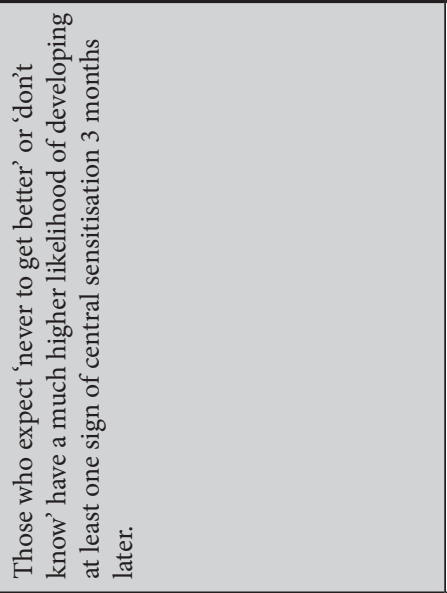 & 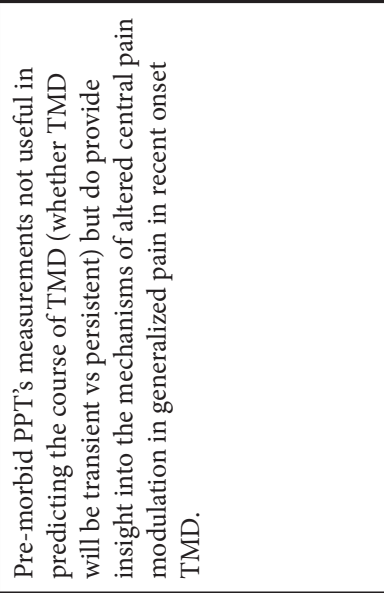 \\
\hline & 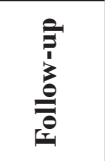 & 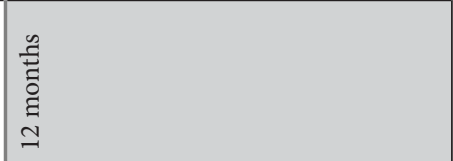 & 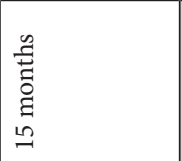 & 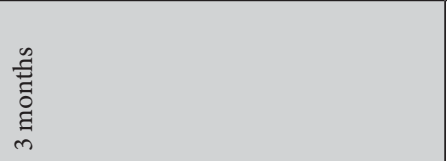 & 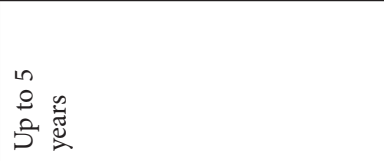 \\
\hline & 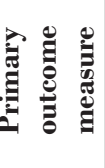 & 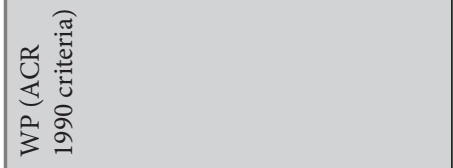 & 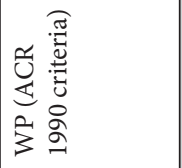 & 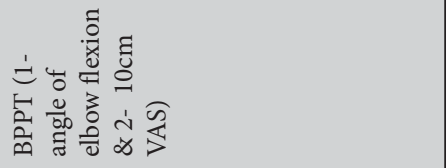 & 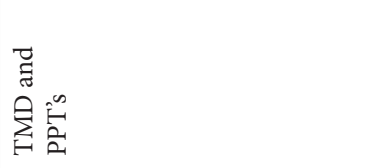 \\
\hline & & 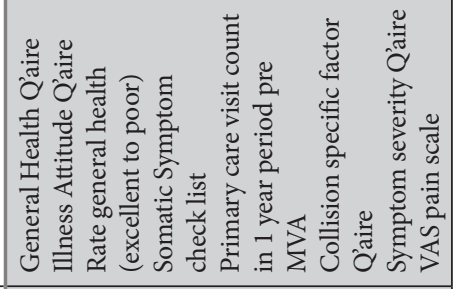 & 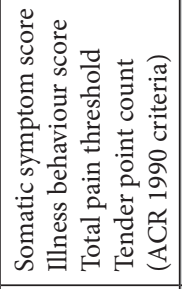 & 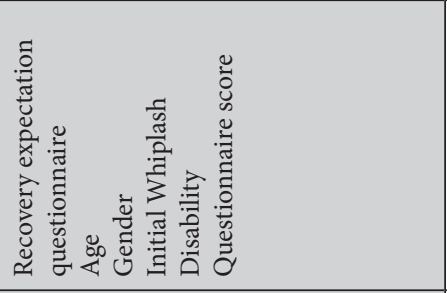 & 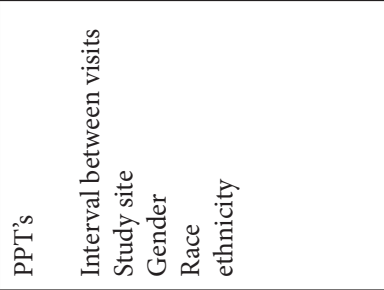 \\
\hline & 产 & 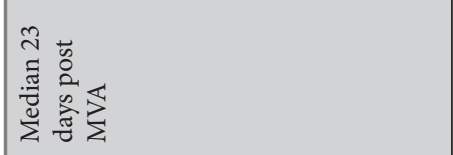 & 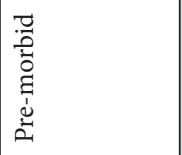 & 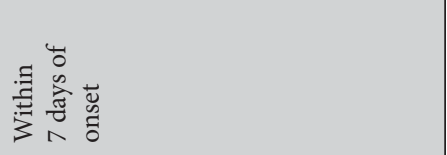 & 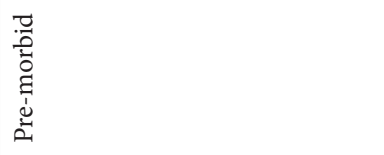 \\
\hline & 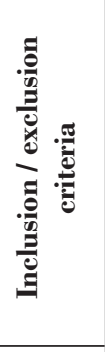 & 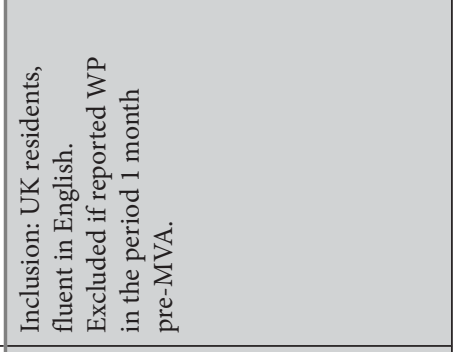 & 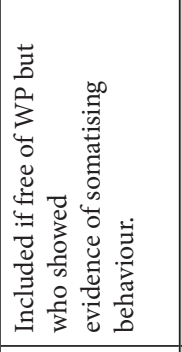 & 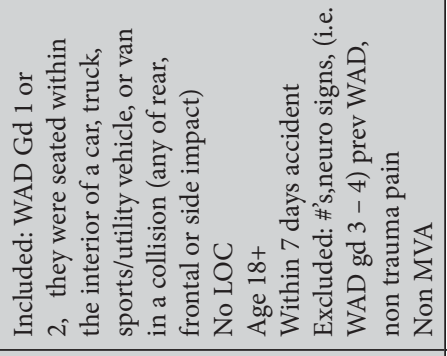 & 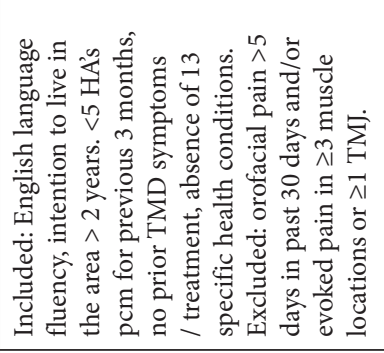 \\
\hline & 苞 & $\hat{\xi}$ & $\xi$ & 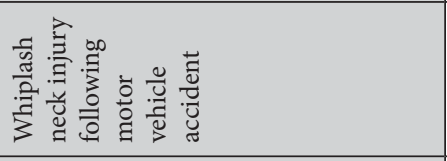 & $\sum_{i}$ \\
\hline & 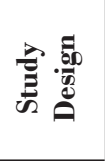 & 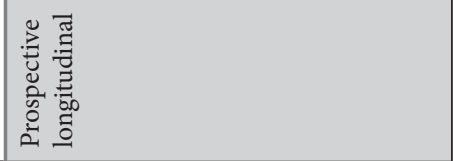 & 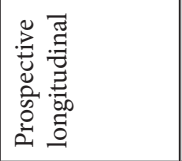 & 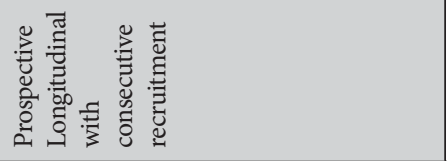 & 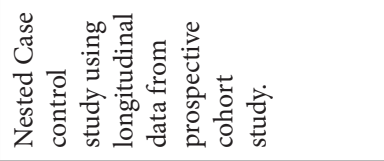 \\
\hline & 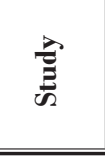 & 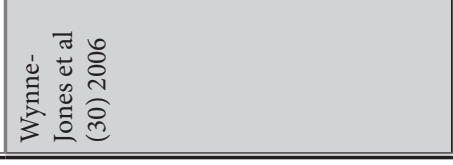 & 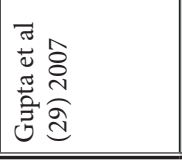 & 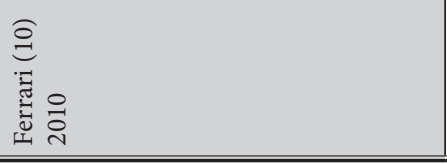 & 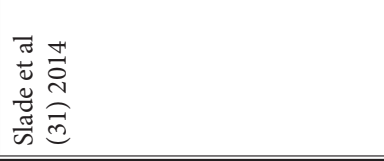 \\
\hline
\end{tabular}




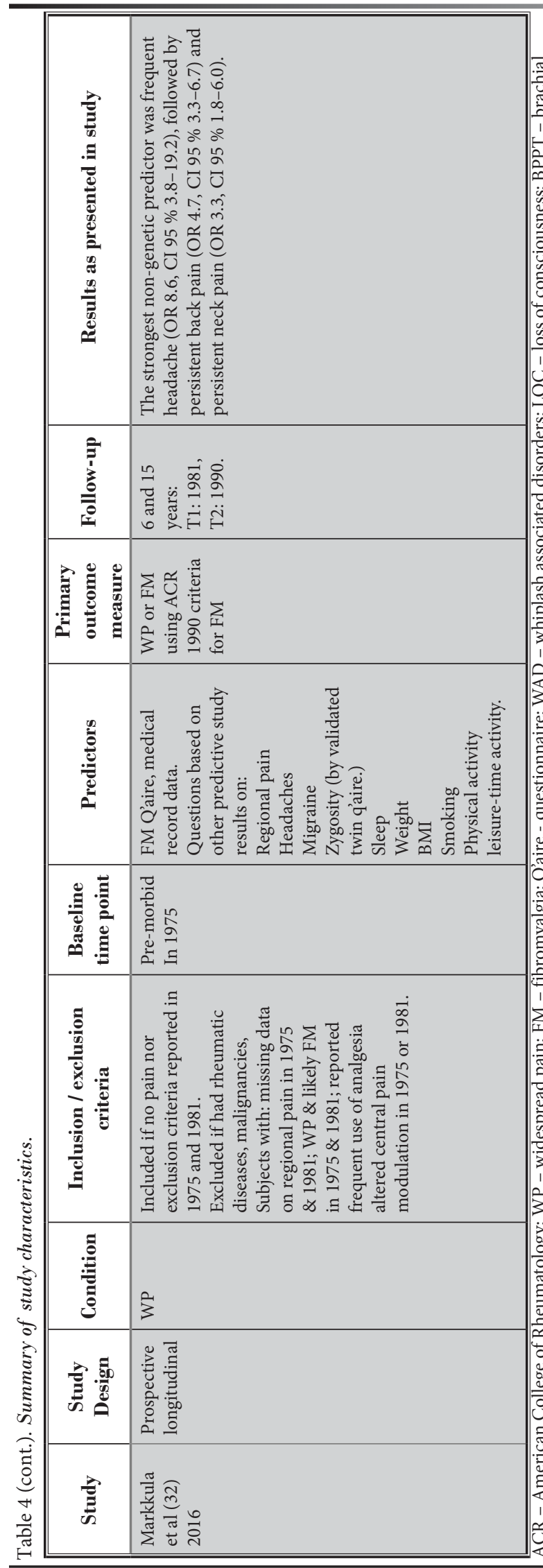

None of the studies selected were specific to LBP, therefore predictors of altered central pain modulation in LBP could not be determined.

\section{Discussion}

This study set out to 1 ) identify predictors of altered central pain modulation in adults with general musculoskeletal pain conditions and secondly, if data were to allow, 2) determine predictors for non-specific low back pain (NSLBP). We found 9 high quality articles, and identified 3 groups of predictors of altered central pain modulation, 2 with a moderate strength of evidence: 1) sensory sensitivity factors, 2) psychological factors; and one which only included one study: 3) other factors.

Some overlapping themes were found, for example, across all studies the musculoskeletal pain conditions were limited to whiplash, temporomandibular disorder (TMD), and WP. Similarly, sensory sensitivity tests were limited to QST, the ACR guidelines (33) and catecholamine-O-methyltransferase (COMT) genetic testing. There was more variation across psychological measures, although the Somatisation Checklist and General Health Questionnaire were used 3 times, enabling some qualitative comparisons. In this review, we did not find any articles that had studied the predictors of altered central pain modulation in NSLBP.

Due to the relatively new concept of altered central pain modulation, in the last 15 years there has been little consensus as to what predictors lead to altered central pain modulation. It is therefore perhaps not surprising that many of the predictors tested varied widely as researchers attempt to narrow down the possibilities. The heterogeneity of predictors and of outcome measures made grouping of factors and outcomes for comparisons broad and prevented meta-analysis of the results.

\section{Definitions of Altered Central Pain Modulation}

One challenge during this review was a lack of definition for altered central pain modulation. At the time of publication of many of the studies, there was a lack of clinical guidelines on how to identify altered central pain modulation in patients. Altered central pain modulation was not directly defined but could be inferred. Some of the studies used the ACR guidelines (33) as a validated measure of WP $(26,27,29,30,32)$. Although the full ACR guidelines provide diagnostic criteria 


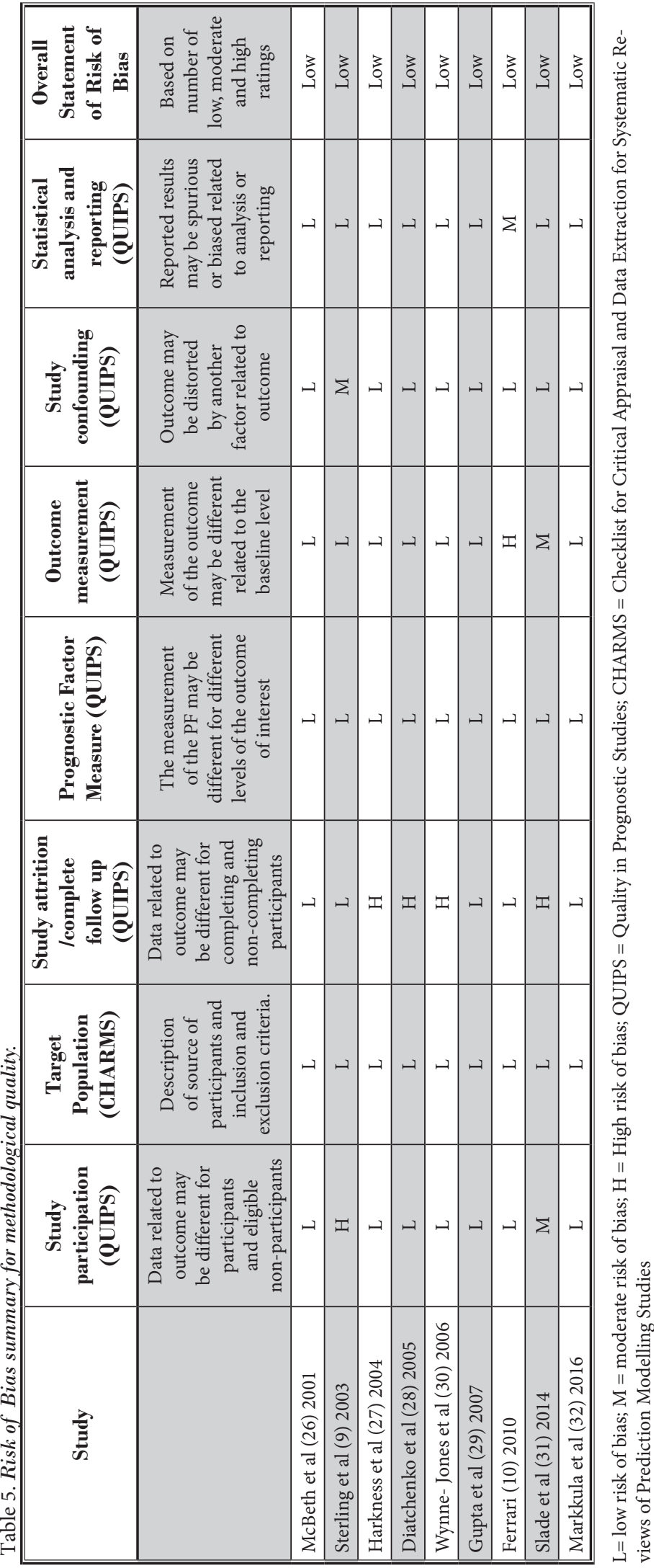

for identifying fibromyalgia, a section of the guidelines specifically identify WP. WP is indicative of altered central pain modulation (7) and is an appropriate primary outcome measure for altered central pain modulation to be included in the current review.

The musculoskeletal pain disorders studied also allowed for inference of altered central pain modulation: whiplash grade 1 or $2(9,10)$, WP $(26,27,29,30,32)$, and TMD $(28,31)$. These musculoskeletal pain disorders, when chronic, have been described as being closely associated with altered central pain modulation $(19,21,34)$ increasing the likelihood that the study populations in the current review contain a proportion presenting with altered central pain modulation at follow-up.

QST was used in 4 studies and included pressure pain thresholds (PPT) $(9,10,28)$, temperature pain thresholds (TPT) (9), and the Brachial Plexus Provocation Test (BPPT) $(9,10)$. While PPTs are a valid measure of altered central pain modulation (20), BPPT, although associated, has not been validated specifically for measuring altered central pain modulation in Ferrari (10). Despite this, the BPPT has been accepted for use by some authors as a test to indicate central hypersensitivity in whiplash associated disorders (35), enabling these 2 studies $(9,10)$ to be eligible for inclusion into the current review.

\section{Should Baseline Measures Be Taken Pre-morbidly or During the Acute Stage?}

In the current study, it was assumed a priori that taking baseline measurements in the acute stage of injury precedes the onset of altered central pain modulation. Sterling and colleagues (9) argue that acute stage measures may not accurately reflect pre-morbid sensory sensitivity as alterations in central pain modulation may have already taken place. However, it could be argued acute-stage sensitivity measures do give an indication of pre-morbid sensitivity status, because those who developed altered central pain modulation showed higher sensitivity at baseline than the rest of the acute-stage cohort, and remained higher at follow-up (9). 
Table 6. Clinical interpretation of results.

\begin{tabular}{|c|c|c|}
\hline Study & Grouped Results & $\begin{array}{c}\text { Quality of } \\
\text { evidence, } \\
\text { strength of } \\
\text { recommendation } \\
\text { (GRADE score) }\end{array}$ \\
\hline \multicolumn{3}{|c|}{ Sensory Hypersensitivity at baseline } \\
\hline Sterling et al (9) (2003) & $\begin{array}{l}\text { Higher sensory sensitivity (using QST) within } 4 \text { weeks of a whiplash injury is a predictor of } \\
\text { altered central pain modulation (low PPT) at } 6 \text { months, associated with moderate to severe } \\
\text { pain and disability and poor recovery. } \\
\text { High sensory sensitivity at the acute stage is apparent in all individuals who experienced a } \\
\text { whiplash injury but sensory sensitivity is 1) less elevated at baseline and 2) returns to normal, } \\
\text { in those who do not develop altered central pain modulation at } 6 \text { months, compared with } \\
\text { those who do. }\end{array}$ & \multirow{4}{*}{ Moderate } \\
\hline $\begin{array}{l}\text { Diatchenko et al (28) } \\
\text { (2005) }\end{array}$ & $\begin{array}{l}\text { Genetic sensitivity to pain, associated with pre-morbid pain sensitivity to QST is a predictor of } \\
\text { altered central pain modulation (TMD with low PPTs and ischaemic pain thresholds). } \\
\text { In this study group, healthy individuals with genetic markers for sensitivity (COMT } \\
\text { genotyping for HPS haplotypes) developed TMD with altered central pain modulation. }\end{array}$ & \\
\hline Gupta et al (29) (2007) & $\begin{array}{l}\text { A high pre-morbid tender point count is a predictor of altered central pain modulation (WP). } \\
\text { In healthy pain-free individuals who show somatising behaviour (Somatization Check list), } \\
\text { PPTs taken at all } 16 \text { points are summed to make a total PPT score. Of those PPTs, the ones } \\
\text { measuring }<4 \mathrm{~kg} / \mathrm{cm}^{2} \text { are counted as tender points and totalled up per participant. }\end{array}$ & \\
\hline Slade et al (31) (2014) & $\begin{array}{l}\text { After the onset of TMD, pre-morbid low PPTs are a predictor of persistent pain and altered } \\
\text { central pain modulation (low PPT). }\end{array}$ & \\
\hline \multicolumn{3}{|l|}{ Psychological factors } \\
\hline $\begin{array}{l}\text { McBeth et al (26) } \\
\text { (2001) }\end{array}$ & $\begin{array}{l}\text { In a healthy population, those who show evidence of somatization before pain onset are more } \\
\text { likely to experience altered central pain modulation in the form of WP within } 12 \text { months of } \\
\text { showing somatization. }\end{array}$ & \multirow{3}{*}{ Moderate } \\
\hline $\begin{array}{l}\text { Wynne-Jones et al (30) } \\
\text { (2006) }\end{array}$ & $\begin{array}{l}\text { A tendency towards somatization and health seeking behaviour pre-morbidly (Somatization } \\
\text { check list and GHQ), increased perception of initial injury severity (Illness attitudes } \\
\text { questionnaire) severity of initial symptoms (symptom severity questionnaire) and older age all } \\
\text { predict altered central pain modulation (WP) after a whiplash injury. }\end{array}$ & \\
\hline Ferrari (10) (2010) & $\begin{array}{l}\text { Responses of [I expect] 'never to get better' or 'don't know' on the Recovery Expectation } \\
\text { questionnaire are predictors of altered central pain modulation (BPPT with VAS) after } \\
\text { whiplash by } 3 \text { months. }\end{array}$ & \\
\hline \multicolumn{3}{|l|}{ Other factors } \\
\hline $\begin{array}{l}\text { Markkula et al (32) } \\
(2016)\end{array}$ & $\begin{array}{l}\text { In a healthy population, pre-morbid frequent headache, followed by subsequent persistent } \\
\text { regional back or neck pain are predictors of altered central pain modulation (WP). }\end{array}$ & NA \\
\hline
\end{tabular}

$\mathrm{NA}=$ not applicable

Pre-morbid baseline measures were taken in the population-based studies reported in the current review, with the advantage that the predictors were clearly taken prior to the development of altered central pain modulation symptoms. As well as the disadvantage of longer periods needed to reach post-morbid follow-up, longer timeframes may introduce confounders based on demographic and time-dependent co-morbidities. Wynne-Jones and colleagues (30) possibly attempted to overcome this by measuring baseline pre-morbid predictors retrospectively using questionnaires around the time of the whiplash (acute stage). While this is com- mendable, a drawback might have been participant recall bias.

\section{Negative Results}

Gupta et al (29) and Slade et al (31) found premorbid PPTs not to be predictive of new onset altered central pain modulation-related musculoskeletal pain. The study by Gupta et al (29) was underpowered and did not find a significant change from baseline PPTs in order to predict first onset WP within 15 months. This may also have been related to the group being an already-at-risk group, with somatization as an inclusion 
criteria. These participants may have already had lower PPTs than a healthy population, making differences more difficult to detect.

Although Slade et al (31) specifically sought to find predictors of new onset TMD with altered central pain modulation, their results did show that at followup, participants with a lower baseline PPT tended to sensitize more vigorously, developing TMD with even lower PPTs post-morbidly. Those with PPTs closer to normal pre-morbidly and who experienced TMD did not develop persistent symptoms and altered central pain modulation but instead made a full recovery. Therefore, it may be interpreted that individuals with premorbid low PPTs may be at greater risk of developing persistent pain with altered central pain modulation, in a TMD population. This may be generalizable to other altered central pain modulation populations such as whiplash, based on the work of Sterling and colleagues (9). Sterling et al (9) measured baseline PPTs within the acute stage of whiplash injury and found that those with lower baseline PPTs developed persistent pain with altered central pain modulation by 6 months.

There may be a difference between insidious onsets of WP or TMD versus traumatic onset of pain following a motor vehicle accident (MVA). Unfortunately, the 3 studies $(9,10,30)$, where the baseline was during the acute stage following a MVA, used different predictors of altered central pain modulation onset and therefore cannot be grouped to compare with studies including insidious pain onset.

\section{Future Considerations}

Although sensory hyper-sensitivity has been measured as a predictor, other aspects of sensory processing alterations have not been evaluated, such as sensory hypo-sensitivity. Mailis-Gagnon and Nicholson (36) have found sensory hypo-sensitivity to be a feature of a sub-group of fibromyalgia patients and these have not been used as predictors in prognostic studies to date. Measures of QST do not provide a full reflection of sensory alterations or differences because they only measure sensory hyper-sensitivity to particular stimuli.

Genetic markers for sensory sensitivity were discussed in 2 papers - Markkula et al (32) with regard to twins and Diatchenko et al (28) with regard to COMT haplotypes. Both studies discuss the likelihood of genetic predisposition to altered central pain modulation, either insidiously or after the first onset of musculoskeletal pain. It may be proposed, on the basis of the cur- rent findings, that pre-morbid trait sensory sensitivity and psychological characteristics such as coping styles, possibly of partly genetic origin, may predispose to altered central pain modulation, either insidiously or once regional pain is experienced.

\section{Psychological Predisposition}

Ferrari (10) used a one-question questionnaire as a predictor in which expectation of recovery predicted altered central pain modulation in a whiplash group. This is a psychological variable and no baseline physical examination was performed to assess for altered central pain modulation for longitudinal comparison. Three studies $(26,29,30)$ found that a tendency towards somatization pre-morbidly was a predictor of altered central pain modulation. Somatization is said to be a measure of distress and anxiety, manifesting as physical symptoms (37). Pre-morbid anxiety was not assessed in any of the studies; it may be useful to assess for premorbid trait anxiety characteristics in future studies. Distress is a measure of coping styles, none of which were assessed as predictors in any of the studies in the current review. Trait anxiety and coping styles may be an important element in the etiology of altered central pain modulation based on somatization being a predictor in the current review

\section{Predisposition Requires a Trigger before Altered Central Pain Modulation Develops}

It is suggested that if a person is predisposed to altered central pain modulation, there requires a trigger, such as an injury or trauma, to start the transition to altered central pain modulation $(28,32)$. This echoes the observations by Latremolier and Woolf (38) that it is not known why some people tend to sensitize more vigorously after an injury. Markkula and colleagues (32) found that if there was initially some regional pain (back or neck) or headaches, this predicted the transition to altered central pain modulation in the form of WP. What is unknown from that study (32) is whether the regional pain was predominantly nociceptive, which might be an important distinction to make in predicting altered central pain modulation.

\section{Methodological Strengths}

The strengths of this review are based around the methodological rigour and the use of altered central pain modulation-specific inclusion/exclusion criteria. Two independent reviewers carried out the searches 
and a third reviewer was available for discussion. Search terms were piloted on advice from previous authors on searching for prognostic or predictive studies.

Methodological guidelines were followed according to more than one source (13-15). The search strategy included relevant databases without filter limitations, extensive hand searching, and the contacting of a large number of pain researchers in order to include any potential studies. A priori registration of the review was done.

Valid risk of bias and data extraction tools were used $(13,23)$ and strict inclusion/exclusion criteria were developed from current guidelines and literature specific to altered central pain modulation enabling close adherence to the research question.

\section{Methodological Limitations}

Only papers published in English were included, to the exclusion of 2 in German. One paper could not be retrieved. Altered central pain modulation had to be inferred due to the lack of definitions available at the times of publication. Interpretation of the reporting of each study where altered central pain modulation was only inferred presented as a challenge at review level. This careful interpretation was done in order to extract altered central pain modulation-specific information and, despite adhering closely to current altered central pain modulation guidelines, may present as a limitation. A further limitation may be that one eligible study from 1984 could not be retrieved and.

\section{Conclusion}

Nine studies were included in the review to identify predictors of altered central pain modulation in adults with general musculoskeletal pain conditions. We found moderate strength of evidence to suggest that sensory hypersensitivity and somatization pre-morbidly, or higher sensory sensitivity and low expectation of recovery at the acute stage of pain are predictors of altered central pain modulation in some musculoskeletal pain conditions. The implications for this review are that pre-morbid traits of sensory sensitivity and anxiety (somatization) might play a role in the development of altered central pain modulation. Further investigations into pre-morbid characteristics of individuals with altered central pain modulation is warranted. This may help identify risk factors likely to predispose a person with acute musculoskeletal pain to the development of chronic pain with altered central pain modulation.

\section{References}

1. Sterling $M$. Identifying those at risk of developing persistent pain following a motor vehicle collision. ] Rheumatol 2006; 33:838-839.

2. Walton D, Macdermid J, Nielson W, Teasell $R$, Reese $H$, Levesque L. Pressure pain threshold testing demonstrates predictive ability in people with acute whiplash. J Ortho Sports Phys Ther 2011; 41:658-665.

3. Iles RA, Davidson M, Taylor NF. Psychosocial predictors of failure to return to work in non-chronic non-specific low back pain: A systematic review. Occup Environ Med 2008; 65:507-517.

4. Iles RA, Davidson M, Taylor NF, O'Halloran, P. Systematic review of the ability of recovery expectations to predict outcomes in non-chronic non-specific low back pain. J Occup Rehab 2009; 19:25-40.

5. Smart KM, Blake C, Staines A, Thacker $M$, Doody C. Mechanisms-based classifications of musculoskeletal pain: Part 1 of 3: Symptoms and signs of central sensitisation in patients with low back ( \pm leg) pain. Manual Therapy 2012; 17:336-344.

6. Nijs J, Van Houdenhove B, Oostendorp RAB. Recognition of central sensitization in patients with musculoskeletal pain: Application of pain neurophysiology in manual therapy practice. Manual Therapy 2010; 15:135-141.

7. Nijs J, Torres-Cueco R, van Wilgen CP, Girbes EL, Struyf F, Roussel N, van Oosterwijck J, Daenen L, Kuppens K, Vanwerweeen L., Hermans L, Beckwee D, Voogt L, Clark J, Moloney N, Meeus M. Applying modern pain neuroscience in clinical practice: Criteria for the classification of central sensitization pain. Pain Physician 2014; 17:447-457.

8. Baert IAC, Lluch E, Mulder T, Nijs J, Noten S, Meeus M. Does pre-surgical central modulation of pain influence outcome after total knee replacement? A systematic review. Osteoarthritis Cartilage 2015; 2:213-223.

9. Sterling M, Jull G, Vicenzino B, KenardyJ. Sensory hypersensitivity occurs soon after whiplash injury and is associated with poor recovery. Pain 2003; 104:509-517.
10. Ferrari R. Predicting central sensitisation - whiplash patients. Aust Fam Physician 2010; 39:863-866.

11. Vlaeyen JWS, Linton SJ. Fear-avoidance and its consequences in chronic musculoskeletal pain: A state of the art. Pain 2000; 85:317-332.

12. Mogil JS. Pain genetics: Past, present and future. Trends in Genetics 2012; 28:258-266.

13. Moons KGM. de Groot JA, Bouwmeester W, Vergouwe Y1, Mallett S, Altman DG, Reitsma JB, Collins GS. Critical Appraisal and Data Extraction for Systematic Reviews of Prediction Modelling Studies: The CHARMS Checklist. PLoS Nedicine 2014; 11:e1001744.

14. Moher D, Alessandro L, Jennifer MT, Douglas GA. Preferred Reporting Items for Systematic Reviews and Meta-Analyses: The PRISMA Statement. Open Medicine 2009; 3:123-130.

15. Dretzke J, Ensor J, Bayliss S, Hodgkinson J,Lordkipanidze M, Riley RD, Fitzmaurice $\mathrm{D}$, Moore D. Methodological issues and recommendations for systematic reviews 
of prognostic studies: An example from cardiovascular disease. Systematic Reviews 2014; 3:140.

16. Roussel, NA, Nijs, J, Meeus, M, Mylius V, Fayt C, Oostendorp R. Central sensitization and altered central pain processing in chronic low back pain: Fact or myth? Clin J Pain 2013; 29:625-638.

17. Malfliet A, Kregel J, Cagnie B, Kuipers M, Dolphens M, Roussel N, Meeus M, Danneels L, Bramer WM, Nijs J. Lack of evidence for central sensitization in idiopathic, non-traumatic neck pain: A systematic review. Pain Physician 2015; 18:223-236.

18. Field A. Discovering Statistics Using SPSS. Third Edition. Sage Publications Ltd. London, 2009.

19. Yunus MB. Central sensitivity syndromes: A new paradigm and group nosology for fibromyalgia and overlapping conditions, and the related issue of disease versus illness. Semin Arthritis Rheum 2008; 37:339-352.

20. Shy ME, Frohman EM, So YT, Arezzo JC, Cornblath DR, Giuliani MJ, Kincaid JC, Ochoa JL, Parry GJ, Weimer LH. Therapeutics and Technology Assessment Subcommittee of the American Academy of Neurology. Quantitative sensory testing: Report of the Therapeutics and Technology Assessment Subcommittee of the American Academy of Neurology. Neurology 2003; 60:898-904.

21. Mayer TG, Neblett R, Cohen H, Howard KJ, Choi YH, Williams MJ, Perez Y, Gatchel RJ. The development and psychometric validation of the central sensitization inventory. Pain Practice 2012; 12:276-285.

22. Neblett R, Cohen $H$, Choi $Y$, Hartzell MM, Williams M, Mayer TG, Gatchel RJ. The central sensitization inventory (CSI): Establishing clinically significant values for identifying central sensitivity syndromes in an outpatient chronic pain sample. J Pain 2013; 14:438-445.

23. Hayden JA, van der Windt DA, Cartwright JL, Côté $P$, Bombardier C. Assessing bias in studies of prognostic factors. Ann Intern Med 2013; 158:280-286.

24. Guyatt $\mathrm{GH}$, Oxman AD, Kunz, R, FalckYtter Y, Vist GE, Liberati A, Schünemann HJ. Rating quality of evidence and strength of recommendations: GRADE: Grading Quality of Evidence and Strength of Recommendations for Diagnostic Tests and Strategies. BM] 2008; 336:1106-1110.

25. Murphy KA, Cornish RD. Prediction of chronicity in acute low back pain. Arch Phys Med Rehabil 1984; 65:334-337.

26. McBeth J, Macfarlane GJ, Benjamin S, Silman AJ. Features of somatization predict the onset of chronic widespread pain: Results of a large populationbased study. Arthritis \& Rheumatism 2001; 44:940-946.

27. Harkness EF, Macfarlane GJ, Nahit E, Silman AJ, McBeth J. Mechanical injury and psychosocial factors in the work place predict the onset of widespread body pain: A two-year prospective study among cohorts of newly employed workers. Arthritis \& Rheumatism 2004; 50:1655-1664.

28. Diatchenko L, Slade GD, Nackley AG, Bhalang K, Sigurdsson A, Belfer I, Goldman D, Xu K, Shabalina SA, Shagin D, Max MB, Makarov SS, Maixner W. Genetic basis for individual variations in pain perception and the development of a chronic pain condition. Hum Mol Genet 2005; 14:135-143.

29. Gupta A, Silman AJ, Ray D, Morriss R, Dickens C, MacFarlane G), Chiu YH, Nicholl B, McBeth J. The role of psychosocial factors in predicting the onset of chronic widespread pain: Results from a prospective population-based study. Rheumatology 2007; 46:666-671.

30. Wynne-Jones G, Jones GT, Wiles NJ,
Silman AJ, Macfarlane GJ. Predicting new onset of widespread pain following a motor vehicle collision. J Rheumatol 2006; 33:968-974.

31. Slade GD, Sanders AE, Ohrbach R, Fillingim RB, Dubner R, Gracely RH, Bair E., Maixner W, Greenspan J. D. Pressure pain thresholds fluctuate with, but do not usefully predict, the clinical course of painful temporomandibular disorder. Pain 2014; 155:2134-2143.

32. Markkula RA, Kalso EA, Kaprio JA. Predictors of fibromyalgia: A populationbased twin cohort study. BMC Musculoskeletal Disorders 2016; 17:29-40.

33. Wolfe F, Smythe HA, Yunus MB, Bennett RM, Bombardier C, Goldenberg DL, Tugwell P, Campbell SM, Abeles M, Clark P, The American College of Rheumatology 1990 Criteria for the Classification of Fibromyalgia. Report of the Multicenter Criteria Committee. Arthritis Rheum 1990; 33:160-172.

34. Kindler LL, Jones KD, Perrin N, Bennett RM. Risk factors predicting the development of widespread pain from chronic back or neck pain. J Pain 2010; 11:1320-1328.

35. Sterling M. Testing for sensory hypersensitivity or central hyperexcitability associated with cervical spine pain. J Manip Physiol Ther 2008; 31:534-539.

36. Mailis-Gagnon A, Nicholson K. Nondermatomal somatosensory deficits: Overview of unexplainable negative sensory phenomena in chronic pain patients. Current Opinion in Anesthesiology 2010; 23:593-597.

37. Kroenke K, Spitzer RL, deGruy FV, Swindle R. A symptom checklist to screen for somatoform disorders in primary care. Psychosomatics 1998; 39:263-272.

38. Latremoliere A, Woolf CJ. Central sensitization: A generator of pain hypersensitivity by central neural plasticity. J Pain 2009; 10: 895-926. 